\title{
An Analytical Comparison of Inpainting Techniques for Effective Selection
}

\author{
Pooja Singh \\ Student, M.Tech \\ Dept. of computer science and \\ Engineering \\ Graphic Era University \\ Dehradun, India
}

\author{
Manish Sharma \\ Assistant professor \\ Dept. of computer science and \\ Engineering \\ Graphic Era University \\ Dehradun, India
}

\author{
Nupur Chauhan \\ Student, M.Tech \\ Dept. of computer science and \\ Engineering \\ Graphic Era University \\ Dehradun, India
}

\begin{abstract}
Image inpainting is a method that restores or repairs a damaged or corrupted piece of the image or video in undetectable form. This method has a broad range of applications, such as removing scratches in previous photographs, removing large objects, text and creative special effects. The major aim of the inpainting is to change the damaged region in an image. In this paper we provide a comparative analysis of different techniques used for image inpainting. In this paper we discuss various inpainting techniques as texture synthesis inpainting, PDE based inpainting, and exemplar based inpainting.
\end{abstract}

\section{General Terms}

Image inpainting, texture synthesis, Exemplar, image processing, object removal

\section{INTRODUCTION}

Inpainting refers to a process used for filling the missing or corrupted regions of an image using information from the surrounding area .The phrase inpainting is derived from the ancient art of restoring image by professional image restorers in museums etc. the central idea behind the algorithm that has been proposed in the literature is to fill these regions with available information from the surroundings [1].

Essential applications of digital inpainting are:

A. Restoration of ancient paintings for conservation purposes

B. Restoring damaged photographs and films

C. object and text removal in images for special effects

D. Digital zooming and edge-based image coding

Figure $1 \& 2$ shows an example of this task where the duster (manually chosen as the target region) is automatically of the image. The algorithms do this in a way that it looks realistic to human being eye. Details that are hidden completely by the object to be removed cannot be improved by any mathematical technique. So the objective for image inpainting is not to improve the original image but to create some image that has a near resemblance with the original image. Basically it is aim is to obtain a visually plausible interpolation in a region of the image wherever the data are missing or we want to just modify it. It has become standard tool in image and video processing with several applications to image restoration [2].The image inpainting techniques fill holes in image by propagating linear structure into the mark region. [3]

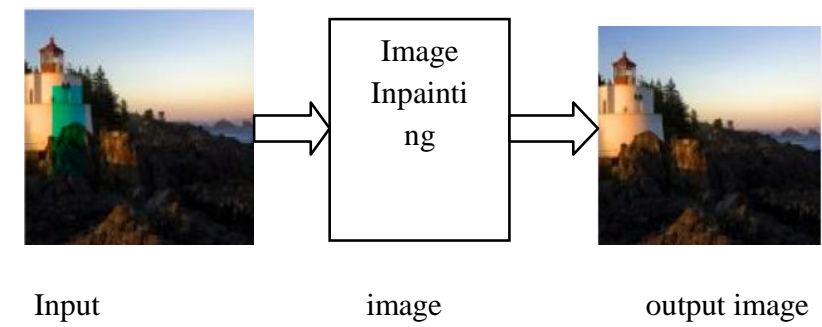

Fig: 1 image inpainting process
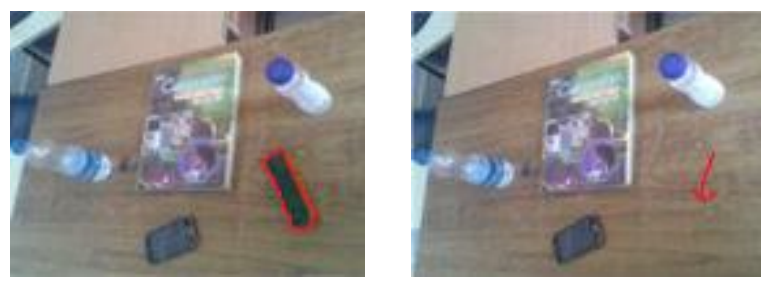

Fig: 2 removing large objects (duster) that have been manually selected using exemplar based inpainting

In image inpainting as to create original image but it is completely not viable without the prior knowledge around the image. It is not likely to reestablish the whole object based on the mandatory existing information. [1]

In this paper various forms of image inpainting techniques presented are discussed.

\section{IMAGE INPAINTING TECHNIQUES}

There are following groups of various image inpainting techniques:

\subsection{Texture synthesis based image inpainting (TSBI)}

Texture synthesis algorithms are commonly used to complete the missing regions using similar neighborhoods of the damaged pixels .In these algorithm an input sample of texture is given and a goal is to produce more of that texture [8].All the earlier inpainting techniques utilized these methods to fill the missing region by sampling and copying pixels from the neighboring area. For e.g. Markov Random Field (MRF) is used to model the local distribution of the pixel and new texture is synthesized by querying existing texture and finding all similar neighborhoods. Their differences exist mainly in 
how continuity is maintained between existing pixels and inpainting hole. [1].These synthesis based techniques perform well only for a select set of images where completing the whole region with homogenous texture information would result in nature completion. This technique works by stitching together small patches of existing images referred to as image quilting. Basically this method can fill big textured regions, however depends on operator selections on sampling position and content.

Texture synthesis approaches divided into three categories:

1) Statistical (parametric)

2) pixel-based (non-parametric)

3) patch-based (non-parametric)

Statistical methods are mostly succeed in reproducing stochastic / irregular textures, but usually fail to reproduce structured/regular textures.

Pixel-based approaches build on the sample texture pixel-bypixel instead of applying filters on it. Their final results are of improved quality than those of statistical methods, but they usually fail to grow large structured textures. Patch-based [9] methods build on a sample texture patch-by-patch as opposed to pixel-by-pixel; therefore they yield faster and more plausible regular textures.

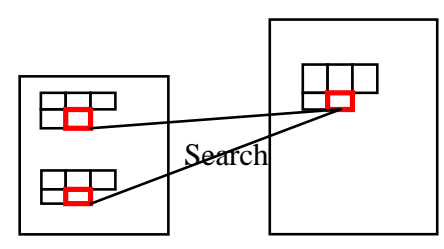

Input

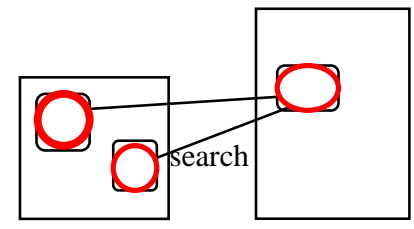

input

\subsection{Exemplar based approach}

Exemplar- based method is a vital class of Inpainting algorithm. This method fills structures in the missing regions using spatial information of neighboring regions. This method is an efficient approach for reconstructing large target regions. Normally, an exemplar-based inpainting algorithm includes the following four main steps:

I. Initializing the Target Region, in which the initial missing areas are extracted and represented with appropriate data structures.

II. Computing Filling Priorities, in this a predefined priority function is used to compute the filling order for all unfilled pixels $p \in \delta \Omega$ in the beginning of each filling iteration.

III. Searching Example and Compositing, in which the most similar example is searched from the source region $\Phi$ to compose the given patch, $\Psi$ (of size $\mathrm{N} \times \mathrm{N}$ pixels) that centered on the given pixel $\mathrm{p}$.

IV. Updating image information in which the boundary $\delta \Omega$ of the target region $\Omega$ and, required information for computing filling priorities are updated.

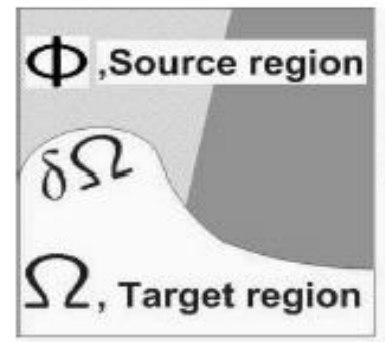

a

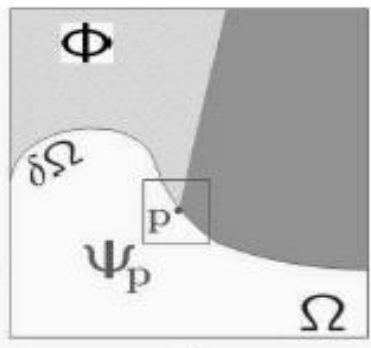

b

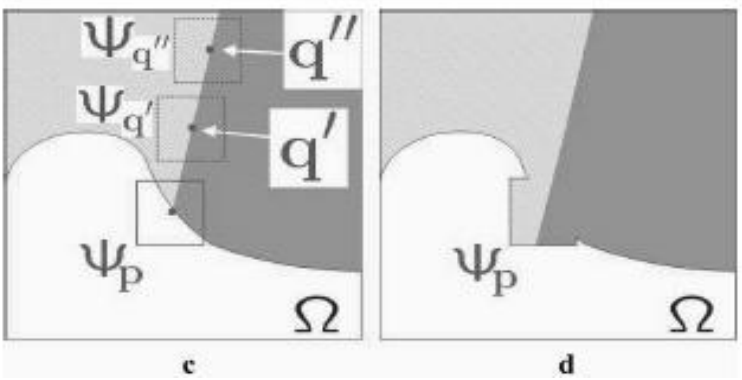

Fig 5: Structure propagation by exemplar-based texture synthesis. (a) Original image, with the mark region $\Omega$, its contour $\delta \Omega$ and the source region $\Phi$ marked. (b) We want to synthesize the area delimited by the patch $\psi p$ centered on the point $p € \delta \Omega$. (c) Most candidate matches for $\psi p$ lie along the boundary between the two textures in the source region, (d) best matching patch in the candidates set has been copied into the position which is occupied by $\psi p$, therefore achieving partial filling[4]

Figure 4: Left: An image with holes (shown in black). Right: the same image reconstructed. 
For the better image quality this algorithm checks the boundary region for sharp changes like edges and assigns additional weights to the unknown pixels nearer to the edges. The algorithm also gives more weights to the pixels near the boundary. And these are accomplished by calculating the edge factor E (p) and known pixels factor K (p) for a patch Pp. centered at pixel p, as shown in Eq3.1 and Eq3.2 respectively

$$
\begin{aligned}
& E(p)=\frac{\sum_{q \in \emptyset \cap P \mathrm{p}} e(q)}{|P \mathrm{p}|} \\
& k(p)=\frac{\sum_{q \in \emptyset \cap P \mathrm{p}} e(q)}{|P \mathrm{p}|}
\end{aligned}
$$

Where $\mathrm{e}(\mathrm{q}), \mathrm{k}(\mathrm{q})$ represents the edges and known pixels in the known area of patch Pp., |Pp.| represents the cardinality of the patch .The $\mathrm{K}$ values of the known and unknown areas are initialized to 1 and 0 respectively. The overall weight is taken as a product of these two terms as in Eq3.3. The pixels with more weight are considered first for filling.

$$
P(p)=E(p) * K(p)
$$

Once a patch is filled the boundary $(\delta \Omega)$ and the weights are recomputed before the next filling. The process is repeated until all pixels of $\Omega$ are complete in. [5]

Exemplar-based inpainting technique uses a patch based sampling and filling, and it has been for a huge part, encouraged by local region-growing methods that grow the texture one pixel or one patch at a time, although maintaining coherence with nearby pixels. Most local pixel-based synthesis techniques depend on Markov random fields (MRFs) modeling of textures. Basically no need of running texture synthesis methods, straight apply to the inpainting problem wherever the known part of the image can be seen as the input texture sample from which the missing pixels can be learned the simple pixel-based texture synthesis technique. As compared with the diffusion based approaches, the exemplar based inpainting achieves a good quality result in recovering textures, and no matter whether they applied into large region or smaller one. Most of the new exemplar based algorithms adopt the greedy strategy, so these algorithms suffer from the ordinary problems of the greedy algorithm [7].

\section{CONCLUSION}

In this paper we review the existing techniques of image inpainting. Here we have provided a detailed description of these techniques, which are used for filling the missing region making use of image. As we know that image inpainting is recently very important research area in the field of image processing. The performances of different techniques are compared as shown in below table on the basis of their uniform area, speed, effectiveness. Most of the algorithms

\begin{tabular}{|c|c|c|c|}
\hline parameters & $\begin{array}{l}\text { Texture } \\
\text { synthesis } \\
\text { based } \\
\text { inpainting } \\
\text { (TSBI) }\end{array}$ & $\begin{array}{l}\text { Partial } \\
\text { differenti } \\
\text { al } \\
\text { equation } \\
\text { (PDE) }\end{array}$ & $\begin{array}{l}\text { Exemplar } \\
\text { based } \\
\text { inpainting } \\
\text { (EBI) }\end{array}$ \\
\hline $\begin{array}{l}\text { Structure } \\
\text { reconstruction }\end{array}$ & $\begin{array}{l}\text { Good for } \\
\text { select set of } \\
\text { images }\end{array}$ & $\begin{array}{l}\text { Good for } \\
\text { smaller } \\
\text { area }\end{array}$ & $\begin{array}{l}\text { Good for } \\
\text { larger area }\end{array}$ \\
\hline $\begin{array}{l}\text { Texture } \\
\text { reconstruction }\end{array}$ & Poor & Poor & Good \\
\hline $\begin{array}{l}\text { Processing } \\
\text { time }\end{array}$ & More & Average & Low \\
\hline $\begin{array}{l}\text { Effectiveness } \\
\text { clarity }\end{array}$ & Not good & Average & Good \\
\hline $\begin{array}{l}\text { Inpaint area } \\
\text { size }\end{array}$ & $\begin{array}{l}\text { Better for } \\
\text { smaller area }\end{array}$ & $\begin{array}{l}\text { Better for } \\
\text { smaller } \\
\text { area }\end{array}$ & $\begin{array}{l}\text { Robust to } \\
\text { change }\end{array}$ \\
\hline $\begin{array}{l}\text { Greedy } \\
\text { approach }\end{array}$ & no & no & yes \\
\hline Application & restoration & $\begin{array}{l}\text { segmentat } \\
\text { ion }\end{array}$ & $\begin{array}{l}\text { Disocculsion } \\
\text {, restoration }\end{array}$ \\
\hline
\end{tabular}
work well only for small area to be inpainted.
Table 1. Comparison between TSBI, PDE and EBI

\section{ACKNOWLEDGMENTS}

The authors acknowledge graphic era university and $\mathrm{Mr}$. Manish Sharma for the contribution and support towards completing this research work.

\section{REFERENCES}

[1]. Marcelo Bertalmio, Luminita Vese, Guillermo Sapiro (2003), Simultaneous Structure and Texture Image Inpainting, IEEE transactions on image processing, vol. 12 , no. 8 ,

[2]. C. Ballester, M. Bertalmio, V. Caselles, G. Sapiro, and J. Verdera, Filling-in by joint interpolation of vector fields and grey levels, IEEE Trans. Image Processing, vol. 10, pp. 1200-1211, Aug. 2001. 
[3]. A. Criminisi, P. P'erez and K. Toyama (2003), Object Removal by Exemplar-Based Inpainting, Microsoft Research Ltd., pp.1-

[4]. A. Criminisi, P. Perez and K. Toyama, Region Filling and Object Removal by Exemplar- Based Image Inpainting, in IEEE Transactions on Image Processing, Vol. 13, No. 9, September 2004

[5] S. Padmavathi, K. P. Soman, Comparative Analysis of Structure and Texture based Image Inpainting Techniques International Journal of Electronics and Computer Science Engineering 1062 ISSN- 2277-1956

[6] T. Chan and J. Shen, Local inpainting models and TV inpainting, SIAM Journal on Applied Mathematics, Vol. 62, 2001, pp. 1019-1043

[7] Komal s Mahajan, Prof. M. B. Vaidya (2012), Image in Painting Techniques: A survey, IOSR Journal of Computer Engineering, Vol.5 (4), pp.45-4
[8] Rajul Suthar, Mr. Krunal R. Patel, a Survey on Various Image Inpainting Techniques to Restore Image, Int. Journal of Engineering Research and Applications ISSN : 2248-9622, Vol. 4, Issue 2( Version 1), February 2014, pp. $85-88$

[9] Li-Yi Wei, Sylvain Lefebvre,Vivek Kwatra, Greg Turk(2009), A State of the Art in Example-based Texture Synthesis, EUROGRAPHICS

[10] V. Janarthanan, G. Jananii (2012), A Detailed Survey on Various Image Inpainting Techniques, International Journal of Advances in Image Processing, Vol. 2, No. 3

[11] J.Y.Wu and Q.Q. Ruan, A novel exemplar based image completion Model (2009), Journal of Information Science and Engineering model, Vol. 25, pp. 481-497.

[12] Z. Xu and S. Jian (2010), Image inpainting by patch propagation using Patch sparsity, IEEE Transactions on Image Processing, Vol.19, and pp. 1153-1165 